\title{
Statistics of Dissipation and Enstrophy Induced by Localized Vortices
}

\author{
Guowei He, ${ }^{1,4, *}$ Shiyi Chen, ${ }^{1,2}$ Robert H. Kraichnan, ${ }^{3}$ Raoyang Zhang, ${ }^{1}$ and Ye Zhou ${ }^{2}$ \\ ${ }^{1}$ Theoretical Division and Center for Nonlinear Studies, Los Alamos National Laboratory, Los Alamos, New Mexico 87545 \\ ${ }^{2}$ IBM Research Division, T. J. Watson Research Center, P.O. Box 218, Yorktown Heights, New York 10598 \\ 3369 Montezuma 108, Santa Fe, New Mexico 87501-2626 \\ ${ }^{4}$ LNM, Institute of Mechanics, Chinese Academy of Sciences, Beijing 100080, People's Republic of China
}

(Received 9 March 1998; revised manuscript received 14 September 1998)

\begin{abstract}
The ratios of enstrophy and dissipation moments induced by localized vorticity are inferred to be finite. It follows that the scaling exponents for locally averaged dissipation and enstrophy are equal. However, enstrophy and dissipation exponents measured over finite ranges of scales may be different. The cylindrical vortex profile that yields maximal moment ratios is determined. The moment ratios for cylindrical vortices are used to interpret differences in scale dependence of enstrophy and dissipation previously found in numerical simulations. [S0031-9007(98)07782-5]
\end{abstract}

PACS numbers: 47.27.Gs, 03.40.Kf, 05.70.Ln, 47.32.Cc

Recent papers on turbulence structure have raised the possibility of different scaling exponents for local averages of enstrophy density and dissipation density, or for longitudinal and transverse structure functions [1-8]. Siggia [9], Kerr [10], and Meneveau et al. [11] have noted that enstrophy is more intermittent than dissipation. More recently, Chen et al. [6] used direct numerical simulation (DNS) data to calculate empirical scalings of enstrophy and dissipation. They found that the exponents for locally averaged enstrophy were significantly smaller than those for locally averaged dissipation. We argue in the present paper that the differences in exponents for enstrophy and dissipation seen over finite ranges of scales cannot survive in the limit of infinite Reynolds number, where the range of scales in the inertial range becomes infinite.

Since large-scale statistics are nearly Gaussian, an obvious implication of different scaling exponents is that, in the limit of infinite Reynolds number, there must exist some vortex structure that supports infinite ratios of enstrophy-density to dissipation-density moments, or of transverse velocity-gradient moments to longitudinalgradient moments. We argue that infinite moment ratios are impossible, in general. The maximal ratios of enstrophy-density to dissipation-density moments are found explicitly for a cylindrical vortex and for a plane vortex layer. The implications of the analysis for the interpretation of DNS and experimental data are discussed.

We deal primarily with enstrophy and dissipation because they are tensor invariants [12]. The arguments are extended to moments of longitudinal and transverse velocity derivatives and differences.

The argument against infinite ratios starts with the observation that a region of fluid with uniform vorticity (Rankin vortex core) is in rigid rotation and suffers zero strain and dissipation. Strain due to differential rotation outside the region makes the all-space enstrophydissipation moment ratios finite, as examined in detail below.
A finite region within which vorticity is confined necessarily exhibits strain and dissipation. Continuity of vorticity requires that the mean vorticity over the region vanish. An elementary identity, given below, states that the changes of vorticity over the region are measured by the changes of strain within the region. From this we infer that the enstrophy-dissipation moment ratios calculated over the volume are finite. Induced dissipation outside the vorticity-containing volume decreases these finite ratios if the averaging volume is extended into the irrotational fluid.

Let $u_{i, j} \equiv \partial u_{i} / \partial x_{j}$ and $u_{i, j m} \equiv \partial^{2} u_{i} / \partial x_{j} \partial x_{m}$. The vorticity and symmetric strain tensors of a solenoidal velocity field $u_{i}(\mathbf{x}, t)$ are

$$
\omega_{i j}=\frac{1}{2}\left(u_{i, j}-u_{j, i}\right), \quad s_{i j}=\frac{1}{2}\left(u_{i, j}+u_{j, i}\right) .
$$

The usual vorticity pseudovector $\boldsymbol{\omega}=\boldsymbol{\nabla} \times \mathbf{u}$ is $\omega_{i}=$ $\epsilon_{i j m} \omega_{j m}$, where $\epsilon_{i j m}$ is the alternating symbol.

The dissipation density (normalized by kinematic viscosity $\nu$ ) and enstrophy density are given by

$$
\epsilon(\mathbf{x})=2 s_{i j}(\mathbf{x}) s_{i j}(\mathbf{x}), \quad \Omega(\mathbf{x})=2 \omega_{i j}(\mathbf{x}) \omega_{i j}(\mathbf{x}) .
$$

The identity

$$
\omega_{i j, m} \equiv s_{m i, j}-s_{m j, i}
$$

is an immediate consequence of $u_{i, j m}=u_{i, m j}$. This identity is the mathematical expression of the physical fact that spatial variation of vorticity is accompanied by strain.

It follows from (3) that the changes in magnitude of vorticity confined within a region are bounded by a linear functional of the changes in strain within the region. For example, the gradient of $\omega_{3}=2 \omega_{12}$ along the $x_{1}$ axis is $\omega_{3,1}=2\left(s_{11,2}-s_{12,1}\right)$. The moments of $\Omega$ and $\epsilon$ over the vorticity-confining volume are given by $\int[\Omega(\mathbf{x})]^{n} d \mathbf{x}$ and $\int[\epsilon(\mathbf{x})]^{n} d \mathbf{x}$. In order for the ratio of these moments to be infinite for some $n$, either $\epsilon(\mathbf{x})$ must vanish throughout the volume or else $\omega(\mathbf{x})$ must have some region of concentration when its value and, hence, 
the nearby values of its gradient are infinite compared to those of $\epsilon$. These possibilities appear to be excluded by (3).

This result extends to cylindrical vortices, which are localized only in the plane normal to the axis. The finite maximal ratios of $\Omega$ moments to $\epsilon$ moments can be obtained explicitly for cylindrical vortices.

A cylindrical vortex is characterized by the azimuthal velocity $v_{\theta}(\mathbf{r})$, where $\mathbf{r}$ is the distance from the vortex axis. The enstrophy density and dissipation density (normalized by $\nu$ ) are

$$
\Omega(r)=\left(\frac{d v_{\theta}}{d r}+\frac{v_{\theta}}{r}\right)^{2}, \quad \epsilon(r)=\left(\frac{d v_{\theta}}{d r}-\frac{v_{\theta}}{r}\right)^{2} .
$$

The enstrophy and dissipation per unit length of vortex are $2 \pi \int_{0}^{\infty} \Omega(r) r d r$ and $2 \pi \int_{0}^{\infty} \epsilon(r) r d r$, respectively. Locally averaged densities may be defined by

$$
\begin{gathered}
\Omega(r, \ell)=\frac{1}{\pi \ell^{2}} \int_{D} \Omega(|\mathbf{x}-\mathbf{r}|) d \mathbf{x}, \\
\epsilon(r, \ell)=\frac{1}{\pi \ell^{2}} \int_{D} \epsilon(|\mathbf{x}-\mathbf{r}|) d \mathbf{x},
\end{gathered}
$$

where $\int_{D}$ is integration, in the plane perpendicular to the vortex axis, over a disk of radius $\ell$ centered on $\mathbf{r}$.

The moments,

$$
\begin{gathered}
\Omega_{n}(\ell)=2 \pi \int_{0}^{\infty}[\Omega(r, \ell)]^{n} r d r, \\
\epsilon_{n}(\ell)=2 \pi \int_{0}^{\infty}[\epsilon(r, \ell)]^{n} r d r,
\end{gathered}
$$

describe the distribution of enstrophy and dissipation densities in the single vortex structure. By $(4), \Omega_{1}(0)=$ $\epsilon_{1}(0)$ if $v_{\theta}(0)=v_{\theta}(\infty)=0$.

Consider first the Rankin vortex of radius $r_{c}$ for which $v_{\theta} \propto r\left(r<r_{c}\right)$ and $v_{\theta} \propto 1 / r\left(r>r_{c}\right)$. Here the vorticity is confined to a rigidly rotating core and all of the dissipation lies outside the core. The ratios $R_{n}=\Omega_{n}(0) / \epsilon_{n}(0)$ calculated from (4) are $R_{n}=2 n-1$.

For the Burgers vortex of radius $r_{c}$,

$$
v_{\theta}(r)=\frac{\Gamma}{2 \pi} \frac{1-\exp \left(-r^{2} / r_{c}^{2}\right)}{r},
$$

where $\Gamma$ is the total circulation. By (4), $\Omega(r) \propto$ $\exp \left(-2 r^{2} / r_{c}^{2}\right)$, and the vorticity and dissipation now overlap. Equation (4) yields $R_{2} \approx 10.65, R_{3} \approx 104.07$, and $R_{4} \approx 1040.02$. To a rough approximation, $R_{n} \sim 10^{n-1}$.

Figure 1 compares the area-weighted radial densities, proportional to $r \Omega(r)$ and $r \epsilon(r)$, for the Rankin and Burgers vortices. In the Rankin vortex, which uniquely expels all dissipation to the exterior of all enstrophy, the degrees of localization of enstrophy and dissipation are much more nearly equal than in the Burgers vortex, which has a region where enstrophy and dissipation overlap. The overlap enables more diffuse dissipation.

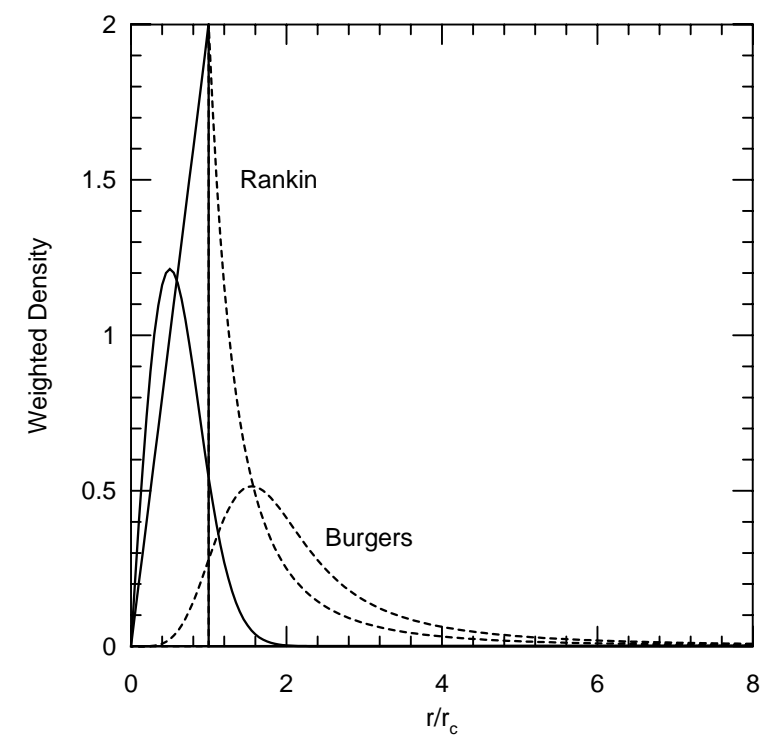

FIG. 1. Enstrophy and dissipation profiles for Rankin and Burgers vortices. The solid lines are $\propto r \Omega(r)$ and the dashed lines are $\propto r \epsilon(r)$. The areas under all four curves are equal.

The cylindrical vorticity distribution that maximizes $R_{2}$ can be found by solving the associated variational problem, with $\int_{0}^{\infty} \omega(r) r d r$ and $\Omega_{1}(0)$ held constant. The result confirms what can be guessed by inspection of (4): The maximizing distribution is the limit $r_{1} / r_{0} \rightarrow \infty$ of

$$
\begin{gathered}
v_{\theta}(r) \propto r \quad\left(r<r_{0}\right), \quad v_{\theta}(r) \propto r^{a} \quad\left(r_{0}<r<r_{1}\right), \\
v_{\theta}(r) \propto 1 / r \quad\left(r>r_{1}\right),
\end{gathered}
$$

with $\alpha=1 / 2$. In this case, $R_{n}=(n-1) 9^{n}$ for $n>$ 1. The essential facts here are first, if $v_{\theta} \propto r^{\alpha}$, then $\Omega(r) / \epsilon(r)$ grows as $\alpha \rightarrow 1$; second, $\alpha=1 / 2$ is the largest $\alpha$ for which $\int_{r_{0}}^{r_{1}}[\Omega(r)]^{2} r d r$ diverges as $r_{0} \rightarrow 0$, thereby making the relative contribution to $\epsilon_{2}$ from the $v_{\theta} \propto 1 / r$ region negligible in the limit. The maximal $R_{n}$ for $n>2$ also are finite; they are maximized at $\alpha$ values that increase with $n$.

The vortex profile that maximizes $R_{2}$ therefore is one in which $\omega(r)$ is highly diffuse and the dominant contribution to $[\Omega(r)]^{2}$ and $[\epsilon(r)]^{2}$ have the same $r$ dependence. This refutes the intuition that the maximizing distribution is one in which a compact vortex core is surrounded by diffuse dissipation.

The ratio $\Omega_{n}(\ell) / \epsilon_{n}(\ell)$ for an axisymmetric vortex equals $R_{n}$ at $\ell=0$, by definition, and approaches unity as $\ell / r_{c} \rightarrow \infty$. The latter property follows from $\Omega_{1}(0)=$ $\epsilon_{1}(0)$. Figure 2 shows $\Omega_{n}(\ell) / \epsilon_{n}(\ell)$, where $n=2$ and $n=4$, for the Burgers vortex (7). The slow decay of the tails of these curves is significant for the interpretation of DNS of turbulence.

The Navier-Stokes (NS) equation has not been invoked in the preceding analysis. Highly diffuse cylindrical vortices are not expected to survive under the vortex stretching and intensification of NS dynamics; the values 


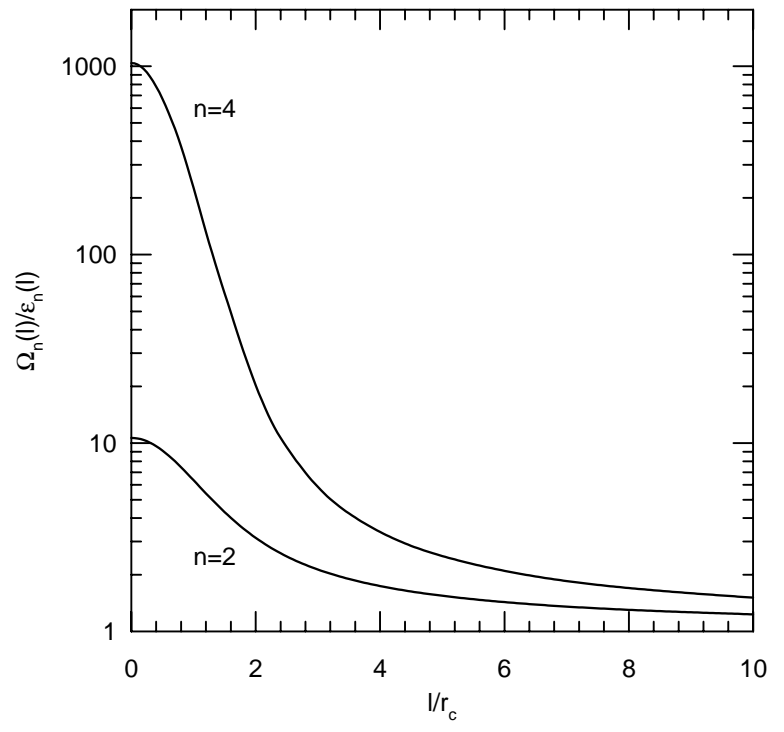

FIG. 2. Moment ratios $\Omega_{n}(\ell) / \epsilon_{n}(\ell)$ for $n=2$ and $n=4$.

$R_{n} \sim 10^{n-1}$ for the Burgers vortex plausibly are the order of the maximal $R_{n}$ attainable under NS.

If the cylindrical vortex is replaced by a plane vortex layer, it is trivial that all $R_{n}=1$. This suggests that the cylindrical vortex may be the form that maximizes the $R_{n}$. Our axisymmetric variational analysis described above can straightforwardly be extended to an arbitrary vorticity distribution in the plane perpendicular to the uniform direction of the vorticity vector. If all the vorticity is confined within a core radius $r_{c}$, then the velocity field and dissipation field at $r \gg r_{c}$ depend only on the total circulation and are independent of details of the vorticity distribution within the core.

The Lundgren-Pullin-Saffman vortex [13] is a tightly wound spiral of unidirectional vorticity. We believe that, for purposes of estimating behavior of moments of enstrophy and dissipation, this structure may be replaced by a strictly axisymmetric profile consisting of concentric shells of vorticity.

By the Biot-Savart law, a compact vortex structure, in which the direction of the vorticity vector is nonuniform, will induce far velocity and far dissipation fields that fall off more rapidly than those of an axisymmetric vortex. Examples are a vortex ring and a vortex dipole consisting of a pair of cylindrical vortices. More rapid falloff of the far dissipation field tends to increase the values of high order dissipation-density moments normalized by mean dissipation.

Consider the moments $\left\langle[\Omega]_{\ell}^{n}\right\rangle,\left\langle[\epsilon]_{\ell}^{n}\right\rangle$, where \langle\rangle denotes ensemble average over a statistically isotropic velocity field and [ ] $e_{e}$ denotes space average over a region of characteristic linear dimension $\ell$. Joint power-law scaling with enstrophy exponents $\zeta_{n}$ and dissipation exponents $\xi_{n}$ exists if there is a range of $\ell$ in which

$$
\left\langle[\Omega]_{\ell}^{n}\right\rangle \propto(\ell / L)^{\zeta_{n}}, \quad\left\langle[\epsilon]_{\ell}^{n}\right\rangle \propto(\ell / L)^{\xi_{n}} .
$$

Here $L$ is a macroscale that marks the bottom of the scaling range. The space averaging [ ] $\ell_{e}$ smears spots of intense excitation. Therefore $\zeta_{n}$ and $\xi_{n}$ are expected to be negative.

Distributions of idealized vortices have been used by a number of authors to model turbulence statistics. The hope is that the vortex distribution can characterize both the essential physics of the small-scale structures and the observed vorticity statistics, thereby leading to an acceptable description of overall turbulence properties. Idealized vortices that have been studied include the Hill vortex [14], simple vortex filaments [15], the Burgers vortex [13,15], and spiral vortices [13]. In [15], an ensemble of Burgers vortices is constructed that yields approximate power-law scaling of structure functions within an inertial range of over a decade.

In actual turbulence and turbulence simulations, the observed vortex structures have a variety of forms. Approximations to compact cylindrical vortices do appear to mediate much of the enstrophy intermittency measured at small scales. Moffatt et al. [16] found that filamentary vortex cores in a DNS contained $63.3 \%$ of the total enstrophy and only $1.3 \%$ of the total dissipation. The strong quasiaxisymmetric vortices have distributions of strengths, core diameters, and core profiles. The distribution of core profiles may be scale dependent. There is also a background of less coherent vorticity and dissipation.

If strong vortices whose $R_{n}$ values are similar to those of a Burgers vortex play an important role, then it is clear from Fig. 2 that substantial differences in the $\ell$ dependence of the flow-wide averages $\left\langle[\Omega]_{\ell}^{n}\right\rangle$ and $\left\langle[\epsilon]_{\ell}^{n}\right\rangle$ are possible. How large the differences actually are, and how closely they may mimic differences in scaling exponents for enstrophy and dissipation over a finite range of $\ell$, depends on the specifics of the flow statistics.

It must be stressed that the finiteness of the $R_{n}$ implies equal scaling exponents for enstrophy and dissipation at infinite Reynolds number, but does not require equality of approximate scaling exponents measured over finite ranges of scales. This is because the maximal measured moment ratios are what our results confront, and the differences in these ratios induced by differing finiterange exponent differences may be small.

The high-resolution DNS reported by Chen et al. [6] illustrates this point. The Taylor microscale Reynolds number is 216. Let $\left\langle\Omega^{n}\right\rangle=\left\langle[\Omega]_{\ell=0}^{n}\right\rangle$ and $\left\langle\epsilon^{n}\right\rangle=\left\langle[\epsilon]_{\ell=0}^{n}\right\rangle$ represent the single-point moments of enstrophy and dissipation obtained by averaging over the entire flow. The DNS yields $\left\langle\Omega^{4}\right\rangle /\left\langle\Omega^{2}\right\rangle^{2}=179$ and $\left\langle\epsilon^{4}\right\rangle /\left\langle\epsilon^{2}\right\rangle^{2}=34.5$. It is easily verified from (7) that this overall ratio of enstrophy moments is realized by an array of parallel Burgers vortices if the average number density of vortex cores in the plane normal to the axis is $\sim\left(0.06 / r_{c}\right)^{2}$.

Moreover, it follows from the definition of the $R_{n}$, and their values given after (7), that, for an array of 
Burgers vortices, $\left\langle\epsilon^{4}\right\rangle /\left\langle\epsilon^{2}\right\rangle^{2} \sim\left(R_{2}^{2} / R_{4}\right)\left\langle\Omega^{4}\right\rangle /\left\langle\Omega^{2}\right\rangle^{2} \sim$ $0.11\left\langle\Omega^{4}\right\rangle /\left\langle\Omega^{2}\right\rangle^{2}$. The measured ratio of flatnesses $34.5 / 179 \sim 0.19$ is closer to unity than that of the array of Burgers vortices, but similar in magnitude. This observation extends to the measured values $\left\langle\Omega^{6}\right\rangle /\left\langle\Omega^{3}\right\rangle^{2}=7537$ and $\left\langle\epsilon^{6}\right\rangle\left\langle\epsilon^{3}\right\rangle^{2}=1705$. For Burgers vortices $R_{3}^{2} / R_{6} \sim 0.099$ while $1705 / 7537 \sim 0.226$. The measured moment ratios are roughly consistent with a rather sparse spacing of Burgers vortices.

This DNS yields an inertial range extending over a factor of 4 in $\ell$ within which the $\left\langle[\Omega]_{\ell}^{n}\right\rangle$ and $\left\langle[\epsilon]_{\ell}^{n}\right\rangle$ exhibit nearly straight-line dependence on $\ell$ on a $\log$ log plot. The associated enstrophy exponents are larger in absolute value than the dissipation exponents, and the differences increase with $n$. The point that is crucial to the present discussion is that the ratios $\left\langle[\Omega]_{\ell}^{n}\right\rangle /\left\langle[\epsilon]_{\ell}^{n}\right\rangle$ associated with the differences in exponent remain of order unity because the extent of the inertial range is modest. Thus for $n=8 / 3$, the ratio at the small- $\ell$ end of the inertial range is 1.44 . These ratios are rigorously bounded by the single-point ratios $\left\langle\Omega^{n}\right\rangle /\left\langle\epsilon^{n}\right\rangle$. They are also of the order of the large $\ell / r_{c}$ tails of the $\Omega_{n}(\ell) / \epsilon_{n}(\ell)$ ratios for the Burgers vortex if $\ell / r_{c}$ in Fig. 2 is taken as the half-distance between adjacent vortex axes in the idealized parallel-array model invoked above.

Finite-range exponents for transverse and longitudinal structure functions also have different measured values [6]. If the structures that dominate higher moments at dissipation-range scales are localized in space, then our arguments for equality of asymptotic enstrophy and dissipation exponents extend also to transverse and longitudinal structure functions. Space differences go over into gradients at the top of the inertial range, and the relations $u_{i, j m}=u_{i, m j}$ relate the gradients of transverse and longitudinal velocity derivatives within a localized structure. There is a loophole, however. A plane shear layer, which is not a localized structure, has zero longitudinal velocity derivatives. An ensemble of plane shear layers can be constructed to yield infinite asymptotic moment ratios for transverse and longitudinal derivatives, together with different scaling exponents. Such a construction is very implausible dynamically and observationally.

L'vov et al. [12] have pointed out that longitudinal and transverse structure functions must have the same asymptotic scaling exponents if the associated tensor invariants have asymptotically pure scaling: the longitudinal and transverse moments are linear functions of the same invariants. Our arguments suggest that the asymptotic scaling exponents of all of the invariants of a given order must be equal.

It should be noted that inertial-range structure functions involve a different part of the velocity spectrum than fluctuations of dissipation on inertial-range scales. The former is supported by inertial-range wave num- bers while the latter represents modulation, on inertialrange spatial scales, of excitation at dissipation-range wave numbers [17].

We have reached two principal conclusions. First, differing asymptotic scaling exponents for locally averaged enstrophy and dissipation are impossible. Second, models built on cylindrical vortices can yield substantial differences in scale dependence of locally averaged enstrophy and dissipation moments because the numerical ratios of single-point enstrophy-density to dissipation-density moments are large. These differences are large enough to account for the apparent differences in scaling exponents for enstrophy and dissipation observed over finite inertial ranges in DNS.

We thank M. Nelkin, K. R. Sreenivasan, E. Titi, and J. Z. Wu for useful discussions.

*Electronic address: ghe@t13.lanl.gov

[1] R. Brachet, Fluid Dyn. Res. 8, 1 (1991); G. R. Ruetsch and M. R. Maxey, Phys. Fluids A 4, 2747 (1992); S. Kida and K. Ohkitani, Phys. Fluids A 4, 1018 (1992); T. Passot, H. Politano, P. L. Sulem, J.R. Angilella, and M. Meneguzzi, J. Fluid Mech. 282, 313 (1995).

[2] B. Dhruva, Y. Tsuji, and K. R. Sreenivasan, Phys. Rev. E 56, R4928 (1997).

[3] J. A. Herweijer and W. Van de Water, Advances in Turbulence, edited by R. Benzi (Kluwer, Dordrecht, 1995), Vol. V, pp. 210-216.

[4] R. Camussi, D. Barbagallo, D. Guj, and F. Stella, Phys. Fluids 8, 1181 (1996); R. Camussi and R. Benzi, Phys. Fluids 9, 257 (1997).

[5] R. A. Antonia and B. R. Pearson, Europhys. Lett. 40, 123 (1997).

[6] S. Chen, K. R. Sreenivasan, M. Nelkin, and N. Cao, Phys. Rev. Lett. 79, 1253 (1997); S. Chen, K. R. Sreenivasan, and M. Nelkin, Phys. Rev. Lett. 79, 2253 (1997).

[7] O. N. Boratav, Phys. Fluids 9, 3120 (1997).

[8] S. Grossmann, D. Lohse, and A. Reeh (to be published).

[9] E. Siggia, J. Fluid Mech. 107, 375 (1981).

[10] R. Kerr, J. Fluid Mech. 153, 31 (1985).

[11] C. Meneveau, K. R. Sreenivasan, P. Kailasnath, and M. S. Fan, Phys. Rev. A 41, 894 (1990).

[12] V.S. L'vov, E. Podivilov, and I. Procaccia, Phys. Rev. Lett. 79, 2050 (1997).

[13] P. G. Saffman and D. I. Pullin, Phys. Fluids, 8, 3072 (1996); D. I. Pullin and P. G. Saffman, Annu. Rev. Fluid Mech. 30, 31 (1998).

[14] J. L. Synge and C. C. Lin, Trans. R. Soc. Can. 37, 45 (1943).

[15] N. Hatakeyama and T. Kambe, Phys. Rev. Lett. 79, 1257 (1997).

[16] H. K. Moffatt, S. Kida, and K. Ohkitani, J. Fluid Mech. 259, 241 (1994).

[17] H. Chen, J. R. Jackson, R. M. Kerr, and R. H. Kraichnan, Phys. Fluids A 1, 1844 (1989), Sect. VI. 